\title{
Haptoglobin-2 variant increases susceptibility to acute respiratory distress syndrome during sepsis
}

\author{
V. Eric Kerchberger, ${ }^{1,2}$ Julie A. Bastarache, ${ }^{1,3,4}$ Ciara M. Shaver, ${ }^{1}$ Hiromasa Nagata, ${ }^{5}$ \\ J. Brennan McNeil, ${ }^{1}$ Stuart R. Landstreet, ${ }^{1}$ Nathan D. Putz, ${ }^{1}$ Wen-Kuang Yu, ${ }^{1,6,7}$ Jordan Jesse, ${ }^{1}$ \\ Nancy E. Wickersham, ${ }^{1}$ Tatiana N. Sidorova, ${ }^{1}$ David R. Janz,${ }^{8}$ Chirag R. Parikh, ${ }^{9}$ Edward D. Siew, ${ }^{10}$ \\ and Lorraine B. Ware ${ }^{1,4}$ \\ 'Division of Allergy, Pulmonary, and Critical Care Medicine, Department of Medicine, ${ }^{2}$ Department of Biomedical \\ Informatics, ${ }^{3}$ Department of Cell and Developmental Biology, and ${ }^{4}$ Department of Pathology, Microbiology and \\ Immunology, Vanderbilt University Medical Center, Nashville, Tennessee, USA. 5Department of Anesthesiology, Keio \\ University School of Medicine, Tokyo, Japan. ${ }^{6}$ Department of Chest Medicine, Taipei Veterans General Hospital, Taipei, \\ Taiwan, ${ }^{7}$ School of Medicine, National Yang-Ming University, Taipei, Taiwan. ${ }^{8}$ Section of Pulmonary and Critical Care \\ Medicine, Louisiana State University School of Medicine, New Orleans, Louisiana, USA. ${ }^{9}$ Division of Nephrology, \\ Department of Medicine, Johns Hopkins University School of Medicine, Baltimore, Maryland, USA. ${ }^{10}$ Division of Nephrology \\ and Hypertension, Department of Medicine, Vanderbilt University Medical Center, Nashville, Tennessee, USA.
}

Acute respiratory distress syndrome (ARDS) is an inflammatory lung disorder that frequently complicates critical illness and commonly occurs in sepsis. Although numerous clinical and environmental risk factors exist, not all patients with risk factors develop ARDS, raising the possibility of genetic underpinnings for ARDS susceptibility. We have previously reported that circulating cell-free hemoglobin (CFH) is elevated during sepsis, and higher levels predict worse outcomes. Excess CFH is rapidly scavenged by haptoglobin (Hp). A common HP genetic variant, $H P 2$, is unique to humans and is common in many populations worldwide. HP2 haptoglobin has reduced ability to inhibit CFH-mediated inflammation and oxidative stress compared with the alternative HP1. We hypothesized that HP2 increases ARDS susceptibility during sepsis when plasma CFH levels are elevated. In a murine model of sepsis with elevated CFH, transgenic mice homozygous for $\mathrm{Hp2}$ had increased lung inflammation, pulmonary vascular permeability, lung apoptosis, and mortality compared with wild-type mice. We then tested the clinical relevance of our findings in 496 septic critically ill adults, finding that HP2 increased ARDS susceptibility after controlling for clinical risk factors and plasma CFH. These observations identify HP2 as a potentially novel genetic ARDS risk factor during sepsis and may have important implications in the study and treatment of ARDS.

as well as a research contract with CSL Behring (current) and past research contracts with Boehringer Ingelheim and Clobal Blood Therapeutics, all unrelated to the current manuscript. CRP is a named inventor on provisional patent number 62/716,465 titled "System and methods for diagnosing acute interstitial nephritis," which is unrelated to the current manuscript.

Copyright: () 2019, American Society for Clinical Investigation.

Submitted: July 9, 2019 Accepted: September 20, 2019 Published: November 1, 2019

Reference information: /CI Insight. 2019;4(21):e131206. https://doi.org/10.1172/jci. insight.131206.

\section{Introduction}

Acute respiratory distress syndrome (ARDS) is an acute inflammatory lung disorder that frequently complicates critical illness and affects over 200,000 adults per year in the United States (1-3). Despite decades of research, currently there are no disease-modifying treatments that reduce ARDS mortality (4-6). The majority of patients with ARDS clinical risk factors do not develop the syndrome, and there is substantial variability in disease severity among patients who do develop ARDS $(4,7)$. This suggests that additional factors, including inheritable genetic factors, may contribute to ARDS susceptibility $(4,8)$.

Sepsis, a clinical syndrome characterized by dysregulated inflammation and organ dysfunction in response to infection, is the most common cause of ARDS $(1-3,9,10)$. Patients with sepsis have alterations in red blood cell (RBC) membrane stability resulting in hemolysis (11-14). Hemoglobin released from RBCs, termed cell-free hemoglobin $(\mathrm{CFH})$, is detectable in the plasma of $80 \%$ of critically ill sepsis patients (15), and higher plasma CFH levels are associated with increased organ dysfunction and mortality during sepsis $(15,16)$. CFH causes organ injury via scavenging of nitric oxide in vascular beds (17), injury to the vascular endothelium (18), neutrophil activation (19), and oxidation of lipid cell membranes $(20,21)$. Therefore, elevated plasma CFH during sepsis may contribute to development of ARDS. 

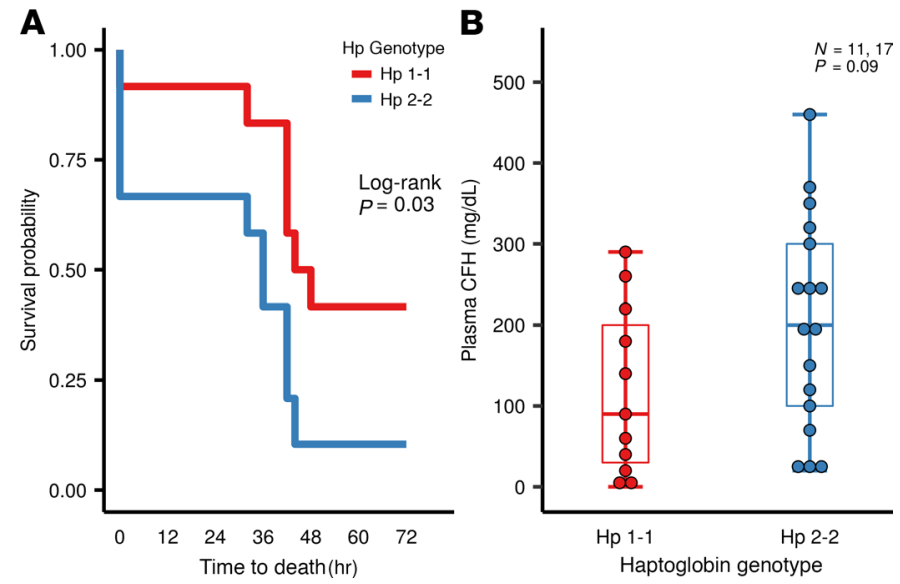

Figure 1. Hp2-2 mice have decreased survival over $\mathbf{7 2}$ hours following experimental sepsis. Hp1-1 (shown in red) and Hp2-2 (shown in blue) mice ( $n=12$ each group) were treated with intraperitoneal injection of $2.0 \mathrm{mg}$ CS per gram body weight and intravenous injection of $100 \mu \mathrm{L}$ of $0.15 \mathrm{mg} / \mathrm{g}$ CFH, then monitored for survival over 72 hours. (A) Survival curves showing survival was significantly worse in $\mathrm{Hp2}-2$ mice. $P=0.03$ by the Mantel-Cox log-rank test. (B) Plasma CFH levels were measured at 24 hours in Hp1-1 $(n=11)$ and Hp2-2 $(n=17)$ mice treated with CS and intravenous (IV) CFH. Dots represent individual values. In the box plots, the thick horizontal bars represent the median, boxes represent the IQR (25th and 75th percentiles), and whiskers represent the minimum and maximum values within $1.5 \times$ IQR from the 25th and 75th percentiles. $P=0.09$ by Mann-Whitney $U$ test.

The plasma protein haptoglobin $(\mathrm{Hp})$ serves as the primary endogenous scavenger for $\mathrm{CFH}$ in mammals (22). $\mathrm{Hp}$ binds irreversibly to $\mathrm{CFH}$, and the resultant $\mathrm{CFH} / \mathrm{Hp}$ complex binds with high affinity to the CD163 receptor present on monocytes and macrophages (23), resulting in endocytosis and clearance of $\mathrm{CFH}$ from the circulation $(24,25)$. Humans have a unique HP genetic variant, HP2 (26), which makes up $45 \%$ of the $H P$ allele frequencies in African American and West African populations, $60 \%$ in European populations, and $75 \%$ in East and South Asian populations (27). Hp from subjects homozygous for the HP2 variant (HP2-2 genotype) has reduced ability to inhibit CFH-mediated inflammation and oxidative stress compared with Hp from subjects homozygous for the alternative allele, $\operatorname{HP1}$ (HP1-1 genotype) $(25,28,29)$. The HP2-2 genotype has been associated with increased risk of atherosclerotic coronary artery disease (30, $31)$, diabetic nephropathy $(32,33)$, and worse outcomes after subarachnoid hemorrhage $(34,35)$.

We hypothesized that the HP2 variant increases susceptibility to ARDS in the setting of sepsis with elevated $\mathrm{CFH}$ because $\mathrm{Hp}$ in patients with the HP2 variant would be predicted to have reduced ability to mitigate $\mathrm{CFH}$-mediated oxidative stress and inflammation. Using transgenic mice with a murine homolog of human $H P 2$, we determined the mechanistic effects of HP2 on acute lung injury in an experimental model of polymicrobial sepsis, finding that $H p 2-2$ mice experienced increased lung inflammation, pulmonary vascular endothelial injury, and mortality compared with wild-type Hp1-1 mice. We then validated our observations in a prospective observational cohort study of septic critically ill adults, finding that the HP2 variant was significantly and independently associated with increased susceptibility to ARDS in humans. These findings identify the $H P 2$ variant as a potentially novel genetic risk factor for ARDS during sepsis.

\section{Results}

Hp2-2 mice have decreased survival during experimental sepsis. We first tested the effect of $H p$ genotype on survival in a murine model of polymicrobial sepsis with elevated $\mathrm{CFH}$ levels. Following injection of intraperitoneal cecal slurry (CS) and intravenous CFH, Hp2-2 mice had decreased survival compared with $H p 1-1$ mice $(P=0.03$ by log-rank test, Figure 1A). Although median plasma CFH levels were higher in $4 p 2-2$ mice compared with Hp1-1 mice, these differences did not reach statistical significance ( $P=0.09$ by Mann-Whitney $U$ test, Figure 1B).

Hp2-2 mice have increased lung inflammation during experimental sepsis. We next assessed the effect of $\mathrm{Hp}$ genotype on lung inflammation in the mouse polymicrobial sepsis model. Hp2-2 mice had increased lung inflammation compared with $\mathrm{Hp} 1-1$ mice in response to intraperitoneal CS and IV $\mathrm{CFH}$, as evidenced by increased whole-lung myeloperoxidase activity $(P=0.014$, Figure 2A) and CXCL1 mRNA expression $(P=$ 0.022, Figure 2B). Hp2-2 mice also had increased CXCL1 levels in bronchoalveolar lavage (BAL) fluid compared with $H p 1-1$ mice $(P=0.011$, Figure $2 \mathrm{C})$. 

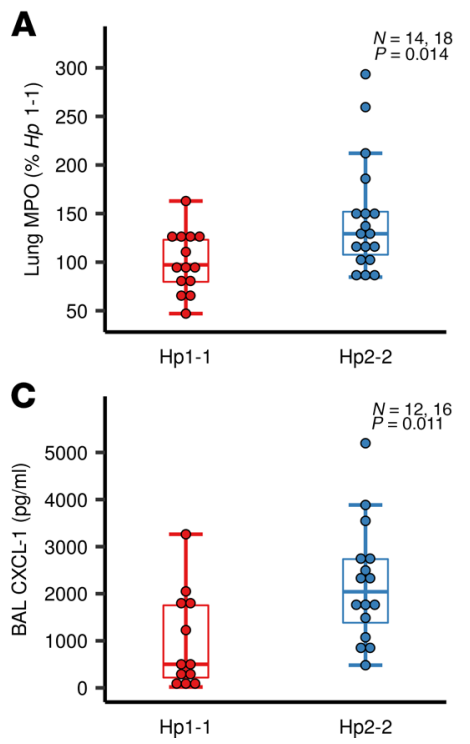

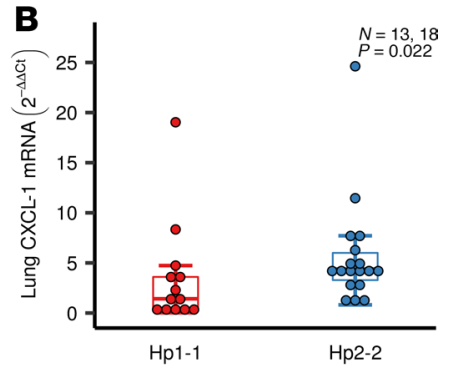

Figure 2. Hp2-2 mice have increased markers of lung inflammation. $\mathrm{Hp1-1}$ (red) and $\mathrm{Hp2-2}$ (blue) mice were treated with CS and IV CFH. (A) Myeloperoxidase (MPO) activity was measured enzymatically in whole lungs. Reported values are normalized to the mean value for Hp1-1 mice. $P=0.014$ by Mann-Whitney $U$ test. (B) Whole-lung mRNA was extracted for CXCL1 and expression measured by real-time PCR. Values are reported as fold-change relative to GAPDH expression. $P=0.022$ by Mann-Whitney $U$ test. (C) CXCL1 protein levels were measured by ELISA in BAL samples. $P=$ 0.011 by Mann-Whitney $U$ test. Dots represent individual values. For the box plots, the horizontal bars represent the median, boxes represent the IQR (25th and 75th percentiles), and whiskers represent the minimum and maximum values within $1.5 \times$ IQR from the 25 th and 75 th percentiles.

Hp2-2 mice have increased pulmonary vascular permeability and lung apoptosis during experimental sepsis. We next tested the effects of $\mathrm{Hp} 2-2$ genotype on the pulmonary vascular endothelium, hypothesizing that $\mathrm{CFH}$ may impair pulmonary vascular barrier function during sepsis. We tested microvascular barrier integrity by retroorbital injection of AngioSense, a $70-\mathrm{kDa}$ near-infrared fluorescent macromolecule that accumulates in sites of increased vascular permeability. Hp2-2 mice had increased lung accumulation of AngioSense compared with Hp1-1 mice 24 hours after intraperitoneal CS and IV CFH ( $P=0.037$, Figure 3 ). This finding indicates that $\mathrm{Hp} 2-2$ mice have worsened pulmonary vascular permeability during sepsis compared with Hp1-1 mice. Excised lungs from Hp2-2 mice also had increased apoptosis by TUNEL staining compared with lungs from $H p 1-1$ mice ( $P=0.004$, Figure 4 ). We have previously determined that $80 \%$ of apoptotic lung cells in this CS + CFH model are endothelial using colabeling in samples from wild-type mice (JA Bastarache et al., unpublished observations). Therefore, this suggests that lung endothelial apoptosis contributes to increased microvascular permeability in this model.

Adult sepsis cohort HP genotyping. To examine the clinical implications of the mechanistic findings from the mouse polymicrobial sepsis model, we tested the association between HP genotype and ARDS in a prospective observational cohort of critically ill adults hospitalized with sepsis. We determined HP genotype by 2 methods depending on data and sample availability. For the 344 patients for whom DNA was available, we directly genotyped HP using real-time PCR. In an additional 152 patients, we used prior GWAS-level genotyping to impute $H P$ genotype using a previously reported algorithm (36). To verify the accuracy of the imputation method in our cohort, we determined genotype by both methods in 120 patients. The observed $H P$ genotype distribution in the entire study cohort $(N=496)$ was $15 \%$ HP1-1, 45\% HP2-1, and 40\% HP2-2 (Supplemental Figure 1; supplemental material available online with this article; https://doi.org/10.1172/jci.insight.131206DS1). The observed $H P$ genotype distribution was similar to expected allele frequencies for a majority-European ancestry cohort (27) ( $P$ $=0.12$ for difference from reference HP2 allele frequency by 2-sample binomial proportions test). The overall agreement between PCR and imputation in the 120 patients genotyped by both methods was 0.91 (Cohen's $\kappa$ 0.85, balanced accuracy 0.91, 95\% CI 0.84-0.95) (Supplemental Table 1 and Supplemental Figure 2).

Adult sepsis cohort patient clinical characteristics. Patients with all $3 \mathrm{HP}$ genotypes had similar baseline clinical characteristics, including age, sex, reported ethnicity, comorbid medical conditions, Acute Physiology and Chronic Health Evaluation II (APACHE II) scores, mechanical ventilation on enrollment, and organ 
A

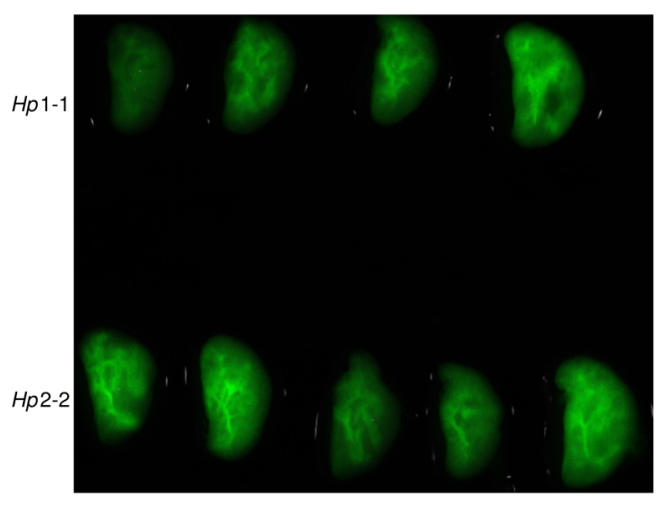

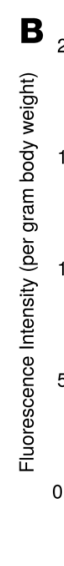

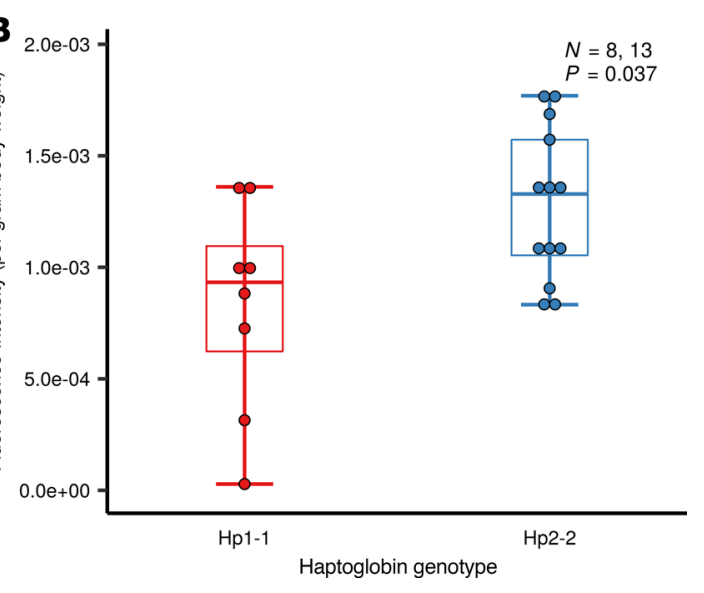

Figure 3. Hp2-2 increases lung microvascular permeability. AngioSense, a fluorescent 70-kD macromolecule, was injected retroorbitally at the time of treatment. (A) After 24 hours, whole lungs were excised and imaged using a high-sensitivity charged coupled device camera. (B) Fluorescent signal per lung was normalized to body weight and quantified. Dots represent individual values. For the box plots, thick horizontal bars represent the median, boxes represent the IQR (25th and 75th percentiles), and whiskers represent the minimum and maximum values within $1.5 \times I Q R$ from the 25 th and 75 th percentiles. $P=0.037$ by Mann-Whitney $U$ test.

failures as measured by Brussels score (37) (Table 1). Plasma CFH levels measured on ICU day 2 were similar across all 3 genotypes, while plasma $\mathrm{Hp}$ levels were significantly decreased in HP2-1 and HP2-2 patients compared with HP1-1 ( $P=0.0008$ by Kruskal-Wallis test, Table 1, Supplemental Figure 3), consistent with prior epidemiological studies of serum $H p$ levels among different $H P$ genotypes $(38,39)$.

Elevated circulating CFH increases risk of ARDS. During the first 4 ICU days, 181 (36.5\%) patients developed ARDS. To test the hypothesis that HP genotype modulates the effect of CFH on ARDS susceptibility, we first tested the association between plasma CFH levels and ARDS susceptibility. We observed a dose-response relationship between plasma $\mathrm{CFH}$ and increased risk for ARDS ( $P=0.032$ by Cochran-Armitage test for increasing ARDS risk by $\mathrm{CFH}$ quartile, Figure 5).

HP2 variant increases ARDS risk in critically ill adults with sepsis. The distribution of ARDS cases among genotypes was HP1-1 28.9\% $(n=22)$, HP2-1 35.3\% $(n=79)$, and HP2-2 40.8\% $(n=80)$. In the unadjusted analysis, ARDS risk significantly increased with increasing number of HP2 alleles. Compared with HP1-1 genotype, HP2-1 had an odds ratio for ARDS of 1.33, and HP2-2 had an odds ratio for ARDS of 1.68, with $P$ $=0.029$ by the Cochran-Armitage test for increasing ARDS risk ordered by number of $H P$ alleles (Figure 6). To test whether the relationship between HP genotype and ARDS was mediated by CFH, we assessed the impact of $H P$ genotype in the presence and absence of detectable $\mathrm{CFH}$. The association between $H P$ genotype and ARDS susceptibility was present only in patients with detectable levels of plasma CFH $(P=0.026$, and $n=414)$ and not in patients with undetectable levels of plasma CFH $(P=0.46$, and $n=82)$ (Figure 7).

$H P 2$ variant is an independent ARDS risk factor. To control for prespecified clinical confounders, we tested the association of $H P$ genotype with ARDS using a multivariable logistic regression model. $H P$ genotype remained independently associated with ARDS risk (odds ratio $=1.41$ per $H P 2$ allele, 95\% CI 1.06-1.88, $P=$ 0.018 ) when controlling for age, sex, ethnicity, severity of illness, plasma $\mathrm{CFH}$, and presence of chronic liver disease (as a surrogate for reduced hepatic Hp synthesis, Table 2, Figure 8). In a sensitivity analysis limited to the 344 patients for whom plasma was available for measurement of Hp levels, a statistically significant association between HP genotype and ARDS risk remained (Supplemental Table 2, Supplemental Figure 4). Additional subgroup analyses restricted to patients with detectable $\mathrm{CFH}$ levels $(n=414, P=0.015)$, of White race $(n=425, P=0.020)$, with absence of chronic liver disease $(n=469, P=0.042)$, and with severe sepsis ( $n=475, P=0.025$ ) also demonstrated a statistically significant association between $H P$ genotype and ARDS risk (data not shown).

HP2 variant and ventilator-free days. To assess whether the HP2 variant affected patient-centered outcomes, we tested the effect of HP genotype on ventilator-free days (VFDs), defined as the number of days alive and not receiving mechanical ventilation from ICU day 1 to ICU day 28 (40). Lower VFD values indicate more prolonged mechanical ventilation, indicating more severe respiratory failure (40). VFDs are a 

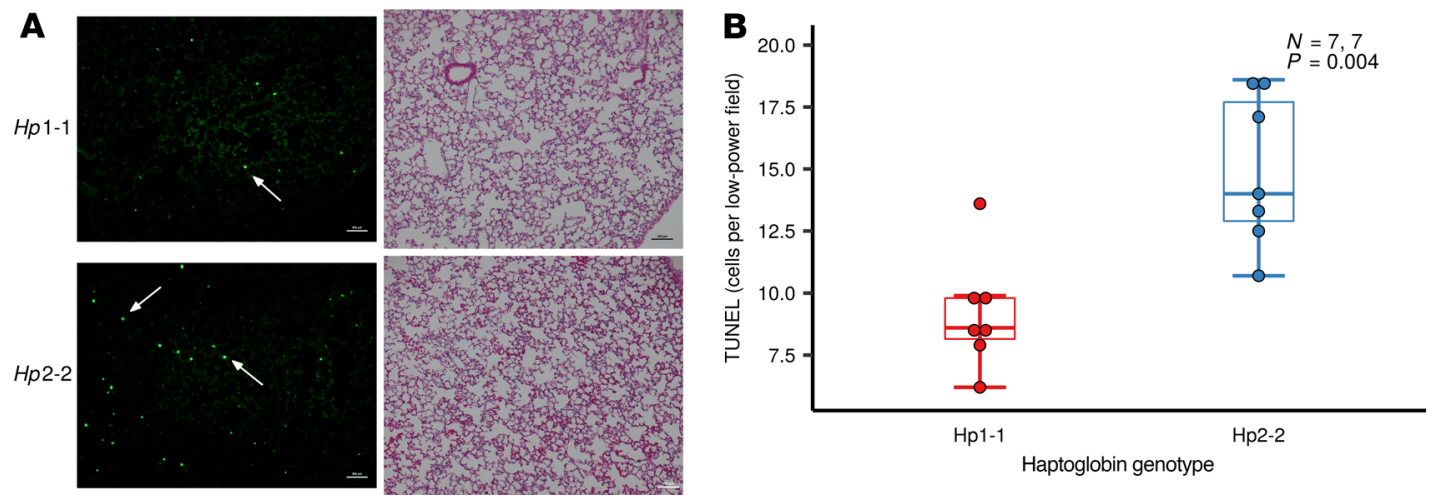

Figure 4. Hp2-2 mice have increased pulmonary apoptosis. Hp1-1 and Hp2-2 mice were treated with CS and IV CFH. Lungs were harvested at 4 hours and examined for apoptotic cells by the TUNEL assay by a trained reviewer blinded to genotype. (A) Representative TUNEL stain images (left images) show increased number of TUNEL-positive cells (white arrows) in Hp2-2 mouse lungs compared with Hp1-1 mouse lungs. H\&E stained sections from the same lung (right images). Scale bars on TUNEL images: $500 \mu \mathrm{m}$, scale bars on H\&E images: $100 \mu \mathrm{m}$. (B) Hp2-2 mouse lungs (blue) demonstrated increased apoptosis of pulmonary cells following sepsis compared with Hp1-1 mice (red). Dots represent individual values. For the box plots, thick horizontal bars represent the median, boxes represent the interquartile range (IQR, 25th and 75th percentiles), and whiskers represent the minimum and maximum values within $1.5 \times$ IQR from the 25 th and 75 th percentiles. ${ }^{*} P=0.004$ by Mann-Whitney $U$ test.

commonly reported outcome in critical care trials because they capture both duration of respiratory failure as well as mortality as a competing outcome (40). Although the number of VFDs decreased with each copy of HP2, with a mean $( \pm$ SEM) of 15.7 ( \pm 2.0 ) days for HP1-1 patients, $14.0( \pm 1.1)$ days for HP 2-1 patients, and 13.5 ( \pm 1.2 ) days for $H P 2-2$ patients, these differences were not significant $(P=0.89$ by Kruskal-Wallis $H$ test, Supplemental Figure 5). We did not observe a difference in in-hospital mortality in ARDS patients between HP genotypes (Supplemental Figure 6) or in 28-day survival (Supplemental Figure 7).

\section{Discussion}

We have identified the HP2 variant as a potentially novel genetic risk factor for ARDS during sepsis. In a mouse polymicrobial sepsis model, $\mathrm{Hp} 2-2$ mice exposed to experimental sepsis had increased lung inflammation, pulmonary vascular injury, and mortality compared with $H p 1-1$ mice. In a cohort of septic critically ill adults, the HP2 variant was independently associated with increased ARDS susceptibility. Moreover, the HP2 variant was associated with increased risk of ARDS only in patients with elevated plasma $\mathrm{CFH}$, supporting a mechanistic role for the $\mathrm{CFH} / \mathrm{Hp}$ axis in ARDS pathogenesis during sepsis. These findings have significant clinical implications because the HP2 variant is more common than HP1 in many populations of European, African, South Asian, and East Asian ancestry (27), potentially affecting over 100,000 ARDS patients per year in the United States alone (1-3).

Endothelial injury with increased microvascular permeability is a defining pathogenic feature of sepsis that leads to shock, organ failure, and death both in human studies and in animal models (41-48). Endothelial injury is also a key pathophysiological characteristic of ARDS $(49,50)$. By comparing Hp2-2 mice with Hp1-1 mice during experimental sepsis, we found that the HP2 variant was associated with increased lung microvascular injury and increased disruption of the alveolar-capillary barrier, in part due to increased apoptosis. The mechanistic impact of HP2 on ARDS during sepsis may be explained by the reduced ability of the HP2 gene product to limit the injurious effects of $\mathrm{CFH}$.

The HP2 variant is a partial copy number variant of $H P 1$ and contains 2 additional exons, one of which encodes a second multimerization domain $(36,51)$. In humans, Hp from individuals with the HP1-1 genotype circulates in plasma as a dimer, whereas Hp from individuals with HP2-1 and HP2-2 genotypes aggregates into progressively larger multimers $(27,28,36,52,53)$. The larger Hp2-2 multimers have reduced ability to prevent $\mathrm{CFH}$-mediated lipid peroxidation compared with Hp1-1 dimers, despite similar binding capacities for CFH $(25,29)$. CFH/Hp2-2 complexes are also cleared more slowly from the extracellular space by CD163 compared with CFH/Hp1-1 complexes (54), despite a greater binding affinity for the CD163 receptor $(23,54)$. Release of $\mathrm{CFH}$ into the circulation during hemolysis represents a significant source of oxidative stress because of the chemical reactivity of the heme iron moiety. Heme-complexed iron can be oxidized from the ferrous $\left(\mathrm{Fe}^{2+}\right)$ state to the more reactive ferric $\left(\mathrm{Fe}^{3+}\right)$ and ferryl $\left(\mathrm{Fe}^{4+}\right)$ states when outside 
Table 1. Demographic and clinical characteristics of study population

\begin{tabular}{|c|c|c|c|c|}
\hline & HP1-1 & HP2-1 & HP2-2 & $\boldsymbol{P}^{\mathrm{A}}$ \\
\hline \multicolumn{5}{|l|}{ Demographics } \\
\hline Number & 76 & 224 & 196 & \\
\hline Male sex & 37 (49\%) & $117(52 \%)$ & $111(57 \%)$ & 0.444 \\
\hline White race & $62(82 \%)$ & $193(86 \%)$ & $170(87 \%)$ & 0.204 \\
\hline \multicolumn{5}{|l|}{ Comorbid medical conditions } \\
\hline Diabetes & $23(30 \%)$ & $75(34 \%)$ & $60(31 \%)$ & 0.778 \\
\hline \multicolumn{5}{|l|}{ ICU characteristics } \\
\hline Mechanical ventilation on enrollment & $47(62 \%)$ & $140(63 \%)$ & $128(65 \%)$ & 0.793 \\
\hline APACHE II Score & $28(7)$ & $28(8)$ & $26(8)$ & 0.066 \\
\hline In-Hospital mortality & $15(20 \%)$ & $49(22 \%)$ & $42(21 \%)$ & 0.925 \\
\hline Coagulation failure & $12(16 \%)$ & $28(13 \%)$ & $23(12 \%)$ & 0.661 \\
\hline Hepatic failure & $15(20 \%)$ & $40(18 \%)$ & $36(18 \%)$ & 0.935 \\
\hline Renal failure & $29(38 \%)$ & $84(38 \%)$ & 72 (37\%) & 0.973 \\
\hline Any organ failure & $65(86 \%)$ & $189(84 \%)$ & $158(81 \%)$ & 0.487 \\
\hline \multicolumn{5}{|l|}{ Plasma biomarkers } \\
\hline Plasma CFH (mg/dL) & $20[10,50]$ & $20[10,30]$ & $20[10,30]$ & 0.251 \\
\hline $\mathrm{Hp}(\mu \mathrm{g} / \mathrm{mL})$ & $1600[800,3880]$ & $1260[570,3300]$ & $860[330,1940]$ & 0.001 \\
\hline \multicolumn{5}{|c|}{$\begin{array}{l}\text { Data are presented as } n(\%) \text { for categorical variables, mean (standard deviation) for continuous variables with normal distributions, and median [IQR] for } \\
\text { continuous variables with non-normal distributions. }{ }^{A} \text { Croupwise comparison testing performed using } \chi^{2} \text { test for categorical variables, } 1 \text {-way ANOVA for } \\
\text { continuous variables with normal distributions, and Kruskal-Wallis } H \text { test for continuous variables with non-normal distributions. }{ }^{B} \text { By Cochran-Armitage } \\
\text { test for trend with alternative hypothesis that risk increases with each HP2 allele. }\end{array}$} \\
\hline
\end{tabular}

of the reducing environment of the RBC cytoplasm (21). Therefore, because Hp2-2 may have less capacity to regulate $\mathrm{CFH}$-mediated oxidative stress, experimental mice with $\mathrm{Hp} 2$ may have increased susceptibility to end-organ injury when plasma CFH levels are elevated during sepsis $(15,55)$

We tested the clinical relevance of our experimental findings in a large prospective observational cohort of critically ill septic adults. The HP2 variant independently associated with increased ARDS susceptibility, with an odds ratio for ARDS of 1.41 per HP2 allele after controlling for clinical factors and plasma CFH. Furthermore, HP genotype affected ARDS susceptibility only in the subgroup of patients with elevated plasma CFH. This finding extends our prior observation that higher $\mathrm{CFH}$ levels are associated with increased risk of organ dysfunction and death during sepsis (15). In addition, our findings support the hypothesis that $H P$ genotype and the $\mathrm{CFH} / \mathrm{Hp}$ axis have a mechanistic role in the pathogenesis of ARDS during sepsis, as observed in our experimental animal model studies. Other investigators have reported associations between HP2-2 genotype and increased risk of many chronic diseases, such as atherosclerotic coronary artery disease $(30,31)$, type 2 diabetes $(56)$, and diabetic nephropathy $(32,33)$. Our current study identifies a potentially novel clinical effect of HP2 affecting risk for a common acute clinical illness with high mortality.

The association between the HP2 variant and increased risk of ARDS may be leveraged in several ways to inform ongoing clinical and experimental research in ARDS. Genotyping the HP2 variant in patients with sepsis may be useful for risk stratification, to identify specific subpopulations at increased risk for ARDS. It may also have use in clinical trial enrichment because patients with the HP2 variant may be more likely to benefit from pharmacological agents that target the $\mathrm{CFH} / \mathrm{Hp}$ axis. Furthermore, these findings may affect studies using human $\mathrm{Hp}$ as a therapeutic agent. Human plasma-derived $\mathrm{Hp}$ is approved in Japan for the treatment of severe hemolysis during extracorporeal cardiopulmonary bypass, severe burn injuries, or massive transfusion following trauma (57-61), but this product is not approved in the United States. Additional experimental stud- 


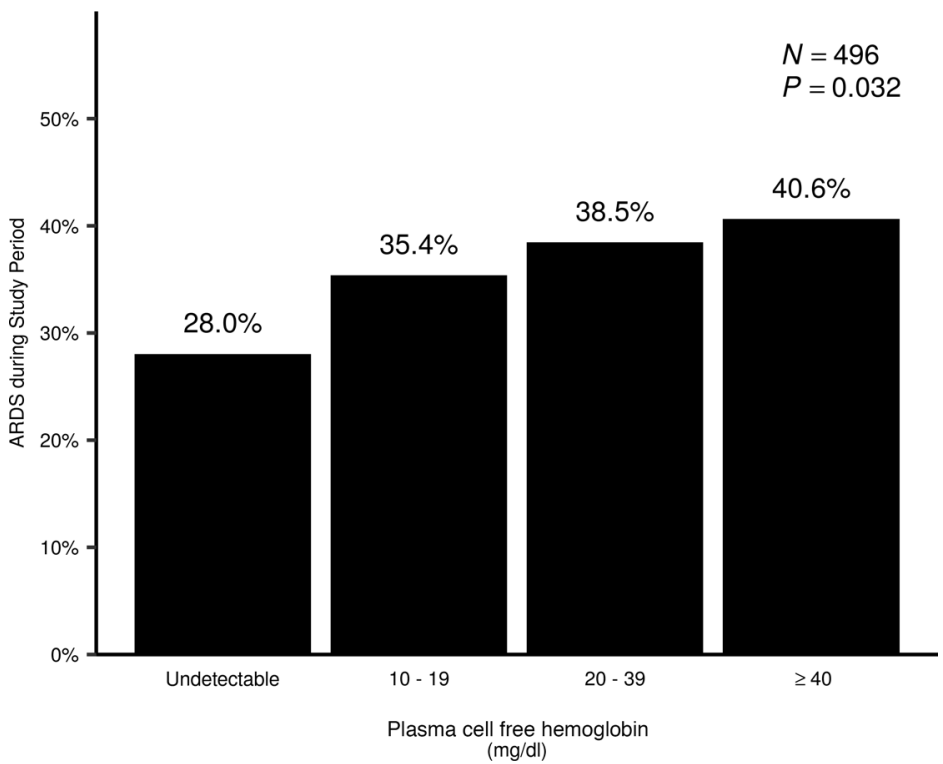

Figure 5. ARDS risk increases with higher plasma CFH during sepsis. Risk of developing ARDS during sepsis increased with higher enrollment plasma $\mathrm{CFH}$ levels. Patients are grouped by enrollment plasma CFH quartile; height of bars and numbers over bars indicate percentage of patients who developed ARDS during the study period. Number of patients per quartile $=82,130$, 156 , and 128, respectively ( $N=496$ in total). $P=0.032$ by Cochran-Armitage test for trend of increasing ARDS risk ordered by CFH quartile. The lower limit of detection for the assay is $10 \mathrm{mg} / \mathrm{dL}$.

ies have reported that $\mathrm{Hp}$ reduces $\mathrm{CFH}$-induced vasoconstriction, hemoglobinuria, and renal dysfunction following massive transfusion $(18,62,63)$; cardiotoxicity following lipopolysaccharide and $\mathrm{CFH}$ administration (64); and organ dysfunction following experimental Staphylococcus aureus pneumonia with massive exchange transfusion (65). These experimental studies used either mixed pooled human $\mathrm{Hp}(18,62)$ or specifically HP2-1 and HP2-2 human Hp (63-65), and the clinical trials used commercial pooled human Hp derived from populations with a high prevalence of HP2-1 and HP2-2 genotypes $(27,57-61)$. Our current study demonstrated differential effects of HP2-2 and HP1-1 genotypes both during experimental murine polymicrobial sepsis and in septic critically ill humans. Therefore, future studies using human $\mathrm{Hp}$ as a novel therapeutic agent may need to consider the Hp phenotype in their study design to maximize clinical benefit.

Our study has several strengths. We used transgenic mice that expressed either the wild-type $H p 1$ or an engineered murine $H p 2$ variant homologous to the human HP2 variant $(66,67)$. This novel murine Hp2 variant has the same size and function as human HP2 (66), thus making it a good model to study the functional effects of $H P$ genotype. Hp2-2 mice exhibit many of the same phenotypes as humans with HP2-2 genotype, including increased coronary atherosclerosis (66) and increased cerebral vasospasm after subarachnoid hemorrhage (68). Given the strong differences we have observed between $H p 2-2$ and $H p 1-1$ mice in response to intraperitoneal sepsis, we propose that this model is ideal to study the molecular mechanisms

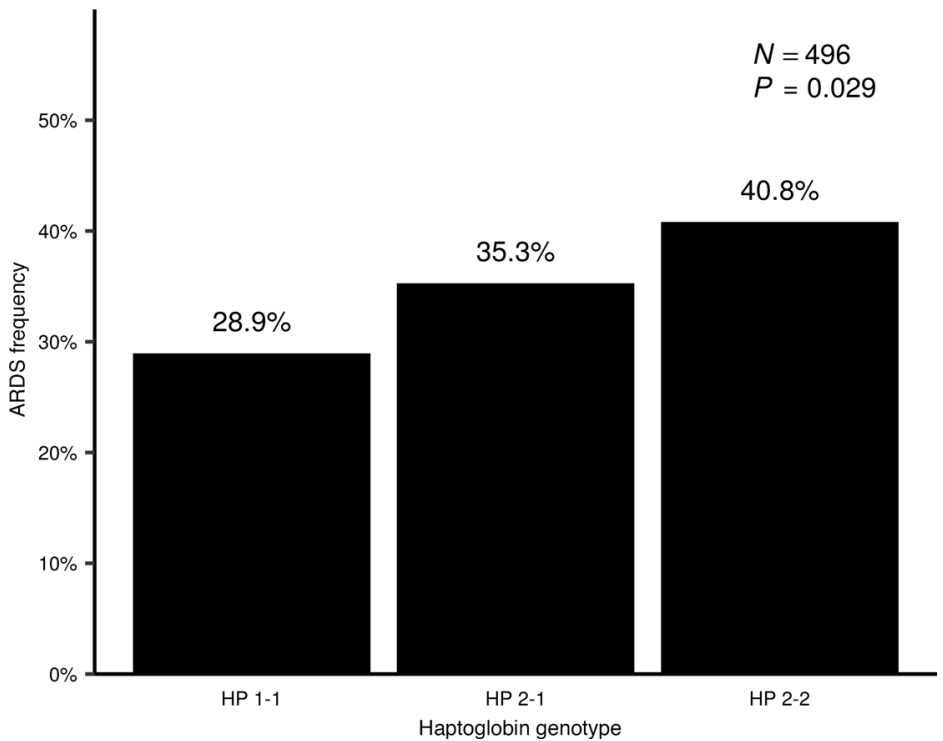

Figure 6. HP genotype increases ARDS risk in septic adults. In the entire study cohort $(N=$ 496), HP2-2 patients and HP2-1 patients had increased risk of developing ARDS during the study period. Height of bars and numbers over bars indicate proportions of patients developing ARDS for each group. $P=0.029$ by Cochran-Armitage test for increasing risk ordered by number of HP2 alleles. 
A

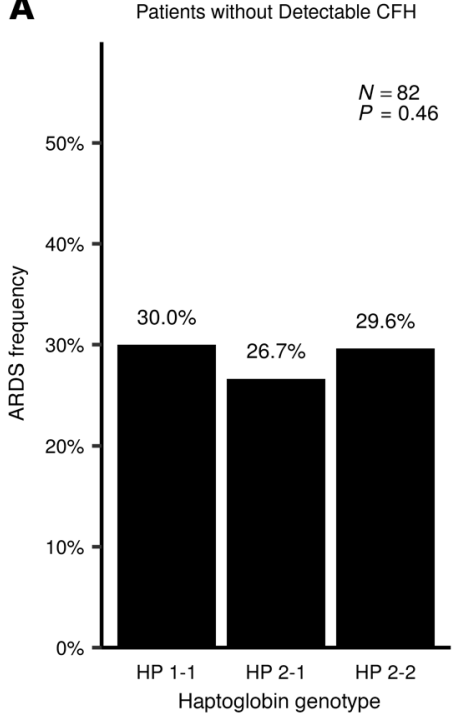

B

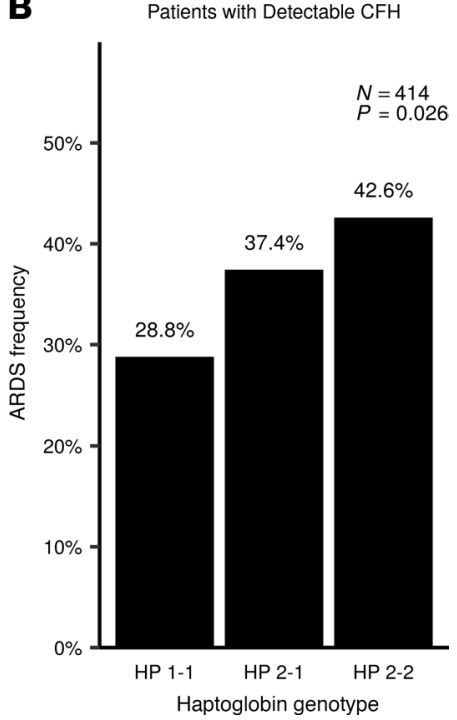

Figure 7. HP genotype affects ARDS risk only in patients with detectable plasma cell-free hemoglobin. (A) There was no association between HP genotype and ARDS risk in patients with undetectable plasma CFH. $n=82$, and $P=0.46$ by Cochran-Armitage test. (B) An association between HP genotype and ARDS risk was observed only in patients with detectable CFH. $n=414$, and $P=$ 0.026 by Cochran-Armitage test.

of the $\mathrm{CFH} / \mathrm{Hp}$ axis in sepsis. Our human cohort study draws from a large well-phenotyped cohort of critically ill adults hospitalized with sepsis. All clinical data were collected prospectively (69). The study was sufficiently sized to determine that the association between the HP2 variant and ARDS risk was not simply due to higher severity of illness or other clinical confounders. Furthermore, the size of the cohort allowed us to test the association between the HP2 variant and ARDS risk by subgroups of patients both with and without detectable plasma $\mathrm{CFH}$, providing further support that $\mathrm{CFH}$ mediates the pulmonary effects of the $H P 2$ variant during sepsis.

Although our study advances understanding of the roles of $\mathrm{Hp}$ and plasma $\mathrm{CFH}$ in the pathogenesis of ARDS, there are some limitations. We do not yet fully understand the mechanisms through which $\mathrm{CFH}$ contributes to risk of ARDS. While the TUNEL staining suggests a role for $\mathrm{CFH}$-induced apoptosis, this assay is nonspecific and can indicate other forms of cell death, such as necroptosis or necrosis (70). Our data suggesting endothelial apoptosis is supported by previous reports showing increased apoptosis of pulmonary microvascular endothelial cells in other murine sepsis models as assessed by caspase activation or flow cytometry (48). We did not examine the role of CD163 in our experimental sepsis model. $\mathrm{CD} 163$ is the primary receptor for monocyte-mediated endocytosis of CFH/Hp complexes (23). Hp2-2 has different binding affinity and clearance kinetics via CD163 compared with Hp1-1 $(23,54)$. Therefore, differential clearance of $\mathrm{CFH} / \mathrm{Hp}$ complexes from the circulation may also contribute to the observed differences in our model between $H p 1-1$ and $H p 2-2$ mice. We also did not examine the role of nitric oxide (NO) in our experimental sepsis model. CFH consumes NO, leading to peripheral vasoconstriction in patients with sickle cell anemia (71), and NO depletion is a common feature of both chronic pulmonary vascular disease (72) and RBC transfusion (17), a known etiology of ARDS (55, 73). Hp binding does not appear to attenuate $\mathrm{CFH}$-mediated $\mathrm{NO}$ scavenging $(62,74)$; however, the slower clearance kinetics of CFH/Hp2-2 complex $(54,74)$ could also affect the rate of NO depletion in the pulmonary vascular

Table 2. Multivariable logistic regression model of ARDS in septic ICU patients

\begin{tabular}{lccc}
\hline Variable & Odds ratio & $\mathbf{9 5 \%}$ Cls & $\boldsymbol{P}$ \\
HP2 allele count & 1.41 & {$[1.06,1.88]$} & 0.018 \\
Age (per 10 years) & 0.76 & {$[0.67,0.87]$} & $<0.0001$ \\
APACHE II (per 5 units) & 1.49 & {$[1.31,1.70]$} & $<0.0001$ \\
Male sex & 1.02 & {$[0.69,1.51]$} & 0.92 \\
Non-White race & 0.52 & {$[0.29,0.95]$} & 0.033 \\
Plasma CFH (per 50 mg/dL) & 1.12 & {$[0.91,1.37]$} & 0.29 \\
Chronic liver disease & 1.62 & {$[0.70,3.74]$} & 0.26
\end{tabular}

Number of subjects: 496. APACHE, Acute Physiology and Chronic Health Evaluation score. 


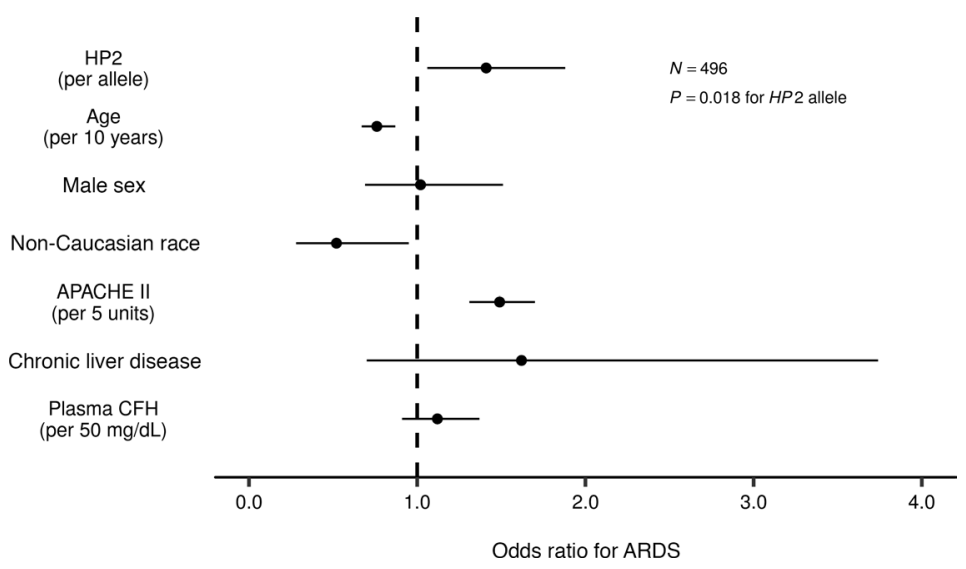

Figure 8. HP2 variant increases ARDS risk in septic adults when controlling for clinical factors. Multivariable logistic regression model for ARDS in study population $(N=496)$. Circles represent point estimate odds ratios, and horizontal lines represent $95 \% \mathrm{Cls}$ for each variable included in the model. Black dashed vertical line indicates odds ratio of 1.0 (no change in ARDS risk). $P=0.018$ for $H P 2$ variant.

endothelium of HP2-2 individuals during sepsis. The cellular mechanisms by which CFH causes disruption of the pulmonary vascular endothelial barrier will require further study. We identified sepsis using the "Sepsis-2" definition (75) because all study patients were enrolled before the publication of the more recent Third International Consensus definition of sepsis (76). Because we enrolled patients exclusively from ICUs, the overwhelming majority $(n=475,95.7 \%)$ of sepsis patients in our cohort also had "severe sepsis" (defined as sepsis with concomitant organ dysfunction or hypoperfusion), which is analogous to the more recent "Sepsis-3" definition (76). We found similar results when limiting our analyses to patients with severe sepsis. Last, although we noted a high correlation between imputed HP genotype and PCR HP genotype in our human study without any evidence of systematic bias, we cannot exclude the possibility of some misclassification among imputed genotypes, particularly between HP2-1 and HP2-2. Reassuringly, we did not observe any misclassification in patients predicted to have HP1-1 by imputation, although the sample size of this group was relatively small.

In summary, we have demonstrated for the first time that the HP2 variant represents a potentially novel genetic risk factor for ARDS susceptibility during sepsis. In an experimental polymicrobial sepsis model, Hp2-2 mice had increased lung inflammation, pulmonary microvascular permeability, lung apoptosis, and death. In critically ill patients with sepsis, each additional HP2 allele was associated with an increased risk of ARDS, independent of potential confounders. These findings have important clinical implications because HP2 is the more common variant in many human populations. This study identifies a large clinical subpopulation of sepsis patients who are genetically predisposed to develop ARDS and has important implications for further research into the role of the $\mathrm{CFH} / \mathrm{Hp}$ axis during critical illness.

\section{Methods}

Transgenic murine model of polymicrobial sepsis. Transgenic mice with a murine homolog of human HP2 (Hp2-2) were a gift from Rafael Tamargo of the Department of Neurosurgery of the Johns Hopkins University School of Medicine (67). We used a previously reported murine model of polymicrobial sepsis $(77,78)$. We prepared a CS from 6-week-old female C57BL/6 donor mice purchased from The Jackson Laboratory (Bar Harbor, Maine, USA). In brief, cecal contents were collected from euthanized donor mice, resuspended in $5 \%$ dextrose at $80 \mathrm{mg} / \mathrm{mL}$, vortexed for 15 seconds, and filtered through a 25 -gauge needle. We administered an intraperitoneal injection CS at $2.0 \mathrm{mg} / \mathrm{g}$ body weight (BW) and a retroorbital injection of $\mathrm{CFH}$ at $0.15 \mathrm{mg} / \mathrm{g} \mathrm{BW}$ to recipient 8 - to 12 -week-old male and female transgenic mice as previously reported (79). The CFH injection was included to increase plasma CFH levels to levels observed in human sepsis (79). We monitored the study mice for 72 hours during survival studies. Mice were monitored closely for signs of pain following induction of experimental sepsis. Antibiotics were not administered in this model of sepsis to allow robust bacterial growth and dissemination, which better reflects the natural history of sepsis in patients before seeking medical care. For all other studies, we euthanized 
the study mice with pentobarbital at 4 or 24 hours after CS administration for collection of samples. The Vanderbilt Institutional Animal Care and Use Committee approved all animal experiments.

Mouse sample collection. Blood was collected by retroorbital puncture in heparinized syringes and centrifuged at $2000 \mathrm{~g}$ for 10 minutes. BAL fluid was collected as previously described (80), as were excised whole lungs, which were immediately flash frozen in liquid nitrogen. We stored all samples at $-80^{\circ} \mathrm{C}$ until the time of analysis.

Plasma circulating CFH in mice. Plasma CFH was measured at 24 hours in mouse plasma using the HemoCue Plasma/Low Hb System (HemoCue America, Brea, California, USA).

Lung inflammation biomarkers in mice. We measured CXCL1/KC in duplicate using an electrochemiluminescence assay (MesoScale Discovery, Gaithersburg, Maryland, USA) in BAL samples according to the manufacturer's recommendations. For mRNA expression, we extracted mRNA from flash-frozen whole lungs using Qiagen RNeasy Plus Mini Kit (Hilden, Germany). We generated cDNA using a SuperScript VILO cDNA Synthesis Kit (Invitrogen, Carlsbad, California, USA) and quantified CXCL1 mRNA expression level by quantitative PCR, normalized to GAPDH expression using TaqMan primer probes (Thermo Fisher Scientific, Waltham, Massachusetts, USA).

MPO activity. Frozen lungs were homogenized in $50 \mathrm{mM}$ potassium phosphate (pH 6.0 ), 0.5\% hexadecyltrimethylammonium bromide, and $5 \mathrm{mM}$ EDTA, then sonicated, centrifuged, and diluted at 1:30 dilution in $100 \mathrm{mM}$ potassium phosphate ( $\mathrm{pH}$ 6.0), 0.3\% hydrogen peroxide (MilliporeSigma, St. Louis, Missouri, USA), and $1 \mathrm{mg} / \mathrm{mL}$-dianisidine (MilliporeSigma). We recorded absorbance at $460 \mathrm{~nm}$ using a spectrophotometer at 1 and 3 minutes and calculated MPO activity according to the equation (81): Activity $=\left(\left[\mathrm{A}_{460 \mathrm{~nm}, 1 \mathrm{~min}}-\mathrm{A}_{460 \mathrm{~nm}, 3 \mathrm{~min}}\right] \times 13.5\right) /$ weight $_{\text {lung }}$

Lung apoptosis. At the time of organ harvest, we perfused lungs with $10 \%$ formalin. Cell apoptosis was ascertained using the Fluorescein In Situ Cell Death Detection Kit (Roche, Basel, Switzerland) on formalin-fixed sections of mouse lung in paraffin following deparaffinization and antigen retrieval. Costaining of endothelial cells was performed by incubating with Mouse Thrombomodulin/BDCA-3 polyclonal goat IgG antibody (AF3894, R\&D Systems, Minneapolis, Minnesota, USA) at 1:200 overnight at $4^{\circ} \mathrm{C}$ and Alexa Fluor 568 anti-goat IgG secondary antibody (A-11057, Invitrogen, Carlsbad, California, USA) at 1:200 for 30 minutes. Lung sections were blinded and 10 non-overlapping images at original magnification $\times 20$ were taken on an Olympus IX51 microscope using an Olympus DP70 camera (Shinjuku, Tokyo, Japan). A trained reviewer who was blinded to each sample's Hp genotype examined lung histology slides and identified the number of TUNEL-positive cells per low-power field. Counts over the 10 fields were averaged for each mouse.

Pulmonary vascular permeability by AngioSense assay. In selected experiments, we administered $100 \mu \mathrm{L}$ AngioSense 750EX near-infrared fluorescent imaging agent ( $2 \mathrm{nmol} / 100 \mu \mathrm{L}$, PerkinElmer, Waltham, Massachusetts, USA) to each study mouse via retroorbital injection. We measured extravascular accumulation of the imaging agent of excised lungs at 24 hours using a LI-COR Pearl small-animal imaging camera (LI-COR Biosciences, Lincoln, Nebraska, USA).

Human study population and clinical data collection. We studied patients greater than 18 years of age enrolled in the Validating Acute Lung Injury markers for Diagnosis (VALID) study, a prospective observational cohort study of critically ill patients at high risk for ARDS and other acute organ dysfunction (69). We included patients with sepsis on admission to the ICU as defined by the American College of Chest Physicians/Society of Critical Care Medicine Consensus ("Sepsis-2") Criteria (75) with plasma samples available for CFH measurement and DNA samples available for genotyping. Sepsis was defined using the Sepsis-2 criteria because all patients included in the study were enrolled before the publication of the Third International Consensus definition of sepsis (76). Study personnel collected clinical data, including medications, vital signs, laboratory studies, severity of illness scores, organ failures by the Brussels criteria (37), and chest radiographs for ARDS phenotyping (82) for the 24 hours before enrollment and daily for the first 4 days after enrollment.

Human sample collection and assays. Study personnel collected blood samples on the morning of ICU day 2 at the time of enrollment and preferentially drew blood through a central venous catheter to minimize hemolysis. We measured CFH (HemoCue Plasma/Low Hb System, HemoCue America, Brea, California, USA) and Hp (Abcam, Cambridge, Massachusetts, USA) in plasma as previously described (15). There was insufficient plasma available to measure Hp levels in 152 patients. We have previously reported some of the hemoglobin and Hp levels $(15,83)$. We extracted genomic DNA from buffy coat peripheral blood leukocytes using Gentra PureGene Blood Kit (Qiagen) according to manufacturer protocols and stored at $-80^{\circ} \mathrm{C}$ until genotyping. 
HP genotyping. We genotyped HP by measuring the ratio of HP5' (a region common to both the HP1 and HP2 variants) to HP2 (a region specific to HP2 variant) via TaqMan-based real-time PCR as previously described (84). We calculated the change in threshold cycle $(\Delta \mathrm{Ct})$ for each sample as $\mathrm{Ct}_{\mathrm{HP5} 5^{\prime}}-\mathrm{Ct}_{\mathrm{HP} 2}$ and calculated the HP2/HP5' ratio of each sample as $2^{-\triangle \mathrm{Ct} \text { sample }}$. We defined HP1-1 by an HP2/HP5' ratio of 0 , HP2- 1 by a ratio between 0 and 0.60 , and HP2-2 by a ratio above 0.60 . We confirmed the accuracy of our PCR genotyping method in a subset of samples with gel electrophoresis to determine Hp phenotype (85) using study personnel blinded to the PCR genotyping results.

HP genotype imputation. In 152 White patients, we imputed HP genotype from genome-wide microarray data. Genotyping was performed at the W.M. Keck facility at Yale University as part of previously reported GWAS of acute kidney injury (86) using the Illumina HumanOmnil Quad v1.0 BeadChip. We used the genotyping v1.9.4 module clustering algorithm from Illumina GenomeStudio software for SNP calling (San Diego, California, USA). Patients with sample genotype call rate under $97 \%$ and patients with a discrepancy between $\mathrm{X}$ chromosome zygosity and reported sex were excluded (86). We extracted SNPs within a 2-Mb region surrounding the $H P$ gene $(\mathrm{Hg} 19 \mathrm{chr16}: 71,036,975-73,063,764)$, then phased the target region and imputed $H P$ genotype using the Beagle version 3.3.2 algorithm (87) with a phased reference panel provided by Boettger et al. (36). The reference panel consisted of 274 unrelated individuals of European ancestry from the 1000 Genomes Project (88) and HapMap3 project (89) who underwent genotyping on several GWAS platforms as well as droplet digital PCR HP genotyping (36). We used the default parameters for Beagle with 50 iterations of the phasing algorithm and 25 haplotypes sampled for each individual during each iteration. We used the calculated genotype posterior probabilities for number of HP2 alleles as the surrogate for HP genotype, with an HP2 genotype probability of $0.0-0.5$ corresponding to a predicted $H P$ genotype of $H P 1-1$, a genotype probability of 0.5-1.5 corresponding to HP2-1, and a genotype probability of 1.5-2.0 corresponding to HP2-2.

Statistics for experimental mouse studies. For the survival study using the experimental sepsis model, we estimated the survival function using the method of Kaplan and Meier and assessed differences in survival using the Mantel-Cox log-rank test. For all biomarker studies, data are presented as the median \pm IQR. We used the Mann-Whitney $U$ test for comparisons of continuous variables between Hp1-1 and Hp2-2 mice.

Statistics for human studies. The primary outcome of the human study was prevalence of ARDS defined by the Berlin Criteria (82) on at least 1 of the first 4 ICU study days. We performed a secondary analysis focused on patients with detectable plasma $\mathrm{CFH}$ levels defined as at least $10 \mathrm{mg} / \mathrm{dL}$ to test the hypothesis that the effect of HP genotype is magnified when plasma CFH levels are elevated. Differences in genotype distributions were tested using the binomial proportion test for observed HP2 allele frequencies. We used the Cochran-Armitage test for trend to test categorical outcomes versus $\mathrm{CFH}$ quartiles and HP genotype, using the alternative hypothesis that risk increases with each HP2 allele. Differences in continuous outcomes between HP genotypes was tested using 1-way ANOVA for variables with normal distributions and the Kruskal-Wallis $H$ test for variables with non-normal distributions. We also tested the association between HP genotype and ARDS using multivariable logistic regression to control for potential clinical and biochemical confounders, including age, sex, ethnicity, severity of illness (by APACHE II score) (90), plasma CFH levels, and presence of chronic liver disease, as a surrogate for hepatic Hp synthetic function. We calculated the number of VFDs during the first 28 days using an accepted definition (40): 0 if the patient died during the first 28 days following enrollment in the study or $28-x$ if the patient was successfully weaned from mechanical ventilation, where $x$ was the number of days receiving mechanical ventilation after enrollment in the study. Categorical outcomes are presented as percentage (number with outcome). Continuous outcomes are presented as mean \pm SEM for outcomes with normal distributions and for VFDs (40) and median \pm IQR for all other non-normally distributed outcomes. A $P$ value of less than 0.05 was considered significant.

To assess the accuracy of imputation-based HP genotyping, we compared imputed $H P$ genotype with PCR-determined HP genotype as the gold standard in 120 patients with data available for both methods. We constructed a $3 \times 3$ confusion matrix and calculated sensitivity, specificity, and $F$ statistics for all 3 possible imputed genotypes. We also calculated overall accuracy and unweighted Cohen's $\kappa$ correlation statistic. We used R version 3.5.1 (91) using the packages DescTools (92) and rms (93) for statistical testing and RStudio version 1.0.147 (94) with the package ggplot2 (95) for data visualization.

Study approval. The Vanderbilt University Medical Center Institutional Animal Care and Use Committee reviewed and approved all animal study protocols. The Vanderbilt University Medical Center Institutional Review Board reviewed and approved the VALID study protocol (IRB 051065). Study personnel 
obtained informed consent from the patient or the patient's surrogate decision maker whenever possible. The IRB approved a waiver of consent when the patient could not give consent because of severity of medical illness and no surrogate decision maker was available.

\section{Author contributions}

VEK, JAB, CMS, and LBW designed the study, provided data analysis and figure generation, performed statistical analyses, and wrote or edited the manuscript. VEK designed and implemented computer scripts for haptoglobin imputation. HN, JBM, SRL, NDP, WKY, JJ, NEW, TNS, and DRJ performed relevant experiments. LBW, CRP, and EDS provided genetic microarray data and performed quality control. All authors reviewed and approved the final version of the manuscript.

\section{Acknowledgments}

Research reported in this publication was supported by the National Institutes of Health (NIH) under award numbers NIH T32GM108554 and T15LM007450 (VEK), NIH R01HL135849 (LBW and JAB), NIH K24HL103836 (LBW), and NIH K08HL136888 (CMS). This work was also supported in part by the Parker B. Francis Foundation (CMS) and Vanderbilt Faculty Research Scholars (CMS). The project publication described was supported by Clinical and Translational Science Awards award UL1TR002243 from the National Center for Advancing Translational Sciences. The authors would like to acknowledge Linda Boettger and Steven McCarroll of the Broad Institute of MIT and Harvard for providing the reference panels for Hp imputation and their advice on implementation, Neil Zheng for assistance with implementation of Hp imputation, and Rafael Tamargo for providing the transgenic mice used in this study. The contents of this project are solely the responsibility of the authors and do not necessarily represent official views of the National Center for Advancing Translational Sciences or the NIH.

Address correspondence to: Lorraine B. Ware, Division of Allergy, Pulmonary, and Critical Care Medicine, Vanderbilt University Medical Center, 1161 21st Avenue South, T-1218, Medical Center North, Nashville, Tennessee, 37232 USA. Phone: 615.322.7872; Email: lorraine.ware@vumc.org.

1. Rubenfeld GD, et al. Incidence and outcomes of acute lung injury. N Engl J Med. 2005;353(16):1685-1693.

2. Rubenfeld GD, Herridge MS. Epidemiology and outcomes of acute lung injury. Chest. 2007;131(2):554-562.

3. Eworuke E, Major JM, Gilbert McClain LI. National incidence rates for Acute Respiratory Distress Syndrome (ARDS) and ARDS cause-specific factors in the United States (2006-2014). J Crit Care. 2018;47:192-197.

4. Thompson BT, Chambers RC, Liu KD. Acute Respiratory Distress Syndrome. N Engl J Med. 2017;377(6):562-572.

5. Bosma KJ, Taneja R, Lewis JF. Pharmacotherapy for prevention and treatment of acute respiratory distress syndrome: current and experimental approaches. Drugs. 2010;70(10):1255-1282.

6. Fan E, Brodie D, Slutsky AS. Acute respiratory distress syndrome: advances in diagnosis and treatment. JAMA. 2018;319(7):698-710.

7. Bellani G, et al. Epidemiology, Patterns of care, and mortality for patients with acute respiratory distress syndrome in intensive care units in 50 countries. JAMA. 2016;315(8):788-800.

8. Meyer NJ, Christie JD. Genetic heterogeneity and risk of acute respiratory distress syndrome. Semin Respir Crit Care Med. 2013;34(4):459-474.

9. Pham T, Rubenfeld GD. Fifty years of research in ARDS. The epidemiology of acute respiratory distress syndrome. A 50th birthday review. Am J Respir Crit Care Med. 2017;195(7):860-870.

10. Doyle RL, Szaflarski N, Modin GW, Wiener-Kronish JP, Matthay MA. Identification of patients with acute lung injury. Predictors of mortality. Am J Respir Crit Care Med. 1995;152(6 Pt 1):1818-1824.

11. Machiedo GW, Powell RJ, Rush BF, Swislocki NI, Dikdan G. The incidence of decreased red blood cell deformability in sepsis and the association with oxygen free radical damage and multiple-system organ failure. Arch Surg. 1989;124(12):1386-1389.

12. Baskurt OK, Gelmont D, Meiselman HJ. Red blood cell deformability in sepsis. Am J Respir Crit Care Med. 1998;157(2):421-427.

13. Piagnerelli M, Boudjeltia KZ, Vanhaeverbeek M, Vincent JL. Red blood cell rheology in sepsis. Intensive Care Med. 2003;29(7):1052-1061.

14. Kempe DS, et al. Suicidal erythrocyte death in sepsis. J Mol Med. 2007;85(3):273-281.

15. Janz DR, et al. Association between cell-free hemoglobin, acetaminophen, and mortality in patients with sepsis: an observational study. Crit Care Med. 2013;41(3):784-790.

16. Adamzik M, Hamburger T, Petrat F, Peters J, de Groot H, Hartmann M. Free hemoglobin concentration in severe sepsis: methods of measurement and prediction of outcome. Crit Care. 2012;16(4):R125.

17. Donadee $\mathrm{C}$, et al. Nitric oxide scavenging by red blood cell microparticles and cell-free hemoglobin as a mechanism for the red cell storage lesion. Circulation. 2011;124(4):465-476.

18. Baek JH, et al. Hemoglobin-driven pathophysiology is an in vivo consequence of the red blood cell storage lesion that can be attenuated in guinea pigs by haptoglobin therapy. J Clin Invest. 2012;122(4):1444-1458. 
19. Graça-Souza AV, Arruda MA, de Freitas MS, Barja-Fidalgo C, Oliveira PL. Neutrophil activation by heme: implications for inflammatory processes. Blood. 2002;99(11):4160-4165.

20. Billings FT, Ball SK, Roberts LJ, Pretorius M. Postoperative acute kidney injury is associated with hemoglobinemia and an enhanced oxidative stress response. Free Radic Biol Med. 2011;50(11):1480-1487.

21. Reeder BJ, Svistunenko DA, Cooper CE, Wilson MT. The radical and redox chemistry of myoglobin and hemoglobin: from in vitro studies to human pathology. Antioxid Redox Signal. 2004;6(6):954-966.

22. Wicher KB, Fries E. Haptoglobin, a hemoglobin-binding plasma protein, is present in bony fish and mammals but not in frog and chicken. Proc Natl Acad Sci USA. 2006;103(11):4168-4173.

23. Kristiansen M, et al. Identification of the haemoglobin scavenger receptor. Nature. 2001;409(6817):198-201.

24. Schaer DJ, Buehler PW, Alayash AI, Belcher JD, Vercellotti GM. Hemolysis and free hemoglobin revisited: exploring hemoglobin and hemin scavengers as a novel class of therapeutic proteins. Blood. 2013;121(8):1276-1284.

25. Gutteridge JM. The antioxidant activity of haptoglobin towards haemoglobin-stimulated lipid peroxidation. Biochim Biophys Acta. 1987;917(2):219-223.

26. Maeda N, Smithies O. The evolution of multigene families: human haptoglobin genes. Annu Rev Genet. 1986;20:81-108

27. Carter K, Worwood M. Haptoglobin: a review of the major allele frequencies worldwide and their association with diseases. Int J Lab Hematol. 2007;29(2):92-110.

28. Okazaki T, Yanagisawa Y, Nagai T. Analysis of the affinity of each haptoglobin polymer for hemoglobin by two-dimensional affinity electrophoresis. Clin Chim Acta. 1997;258(2):137-144.

29. Melamed-Frank M, et al. Structure-function analysis of the antioxidant properties of haptoglobin. Blood. 2001;98(13):3693-3698

30. Levy AP, et al. Haptoglobin phenotype is an independent risk factor for cardiovascular disease in individuals with diabetes: The Strong Heart Study. J Am Coll Cardiol. 2002;40(11):1984-1990.

31. Roguin A, Koch W, Kastrati A, Aronson D, Schomig A, Levy AP. Haptoglobin genotype is predictive of major adverse cardiac events in the 1-year period after percutaneous transluminal coronary angioplasty in individuals with diabetes. Diabetes Care. 2003;26(9):2628-2631.

32. Nakhoul FM, et al. Haptoglobin phenotype and diabetic nephropathy. Diabetologia. 2001;44(5):602-604.

33. Orchard TJ, et al. Haptoglobin genotype and the rate of renal function decline in the diabetes control and complications trial/ epidemiology of diabetes interventions and complications study. Diabetes. 2013;62(9):3218-3223.

34. Ohnishi $\mathrm{H}$, et al. Haptoglobin phenotype predicts cerebral vasospasm and clinical deterioration after aneurysmal subarachnoid hemorrhage. J Stroke Cerebrovasc Dis. 2013;22(4):520-526.

35. Kantor E, et al. Haptoglobin genotype and functional outcome after aneurysmal subarachnoid hemorrhage. J Neurosurg. 2014;120(2):386-390.

36. Boettger LM, et al. Recurring exon deletions in the HP (haptoglobin) gene contribute to lower blood cholesterol levels. Nat Genet. 2016;48(4):359-366.

37. Bernard G. The Brussels Score. Sepsis. 1997;1(1):43-44.

38. Kasvosve I, et al. Reference range of serum haptoglobin is haptoglobin phenotype-dependent in blacks. Clin Chim Acta. 2000;296(1-2):163-170.

39. Imrie $\mathrm{H}$, et al. Haptoglobin levels are associated with haptoglobin genotype and alpha+ -Thalassemia in a malaria-endemic area. Am J Trop Med Hyg. 2006;74(6):965-971.

40. Schoenfeld DA, Bernard GR, ARDS Network. Statistical evaluation of ventilator-free days as an efficacy measure in clinica trials of treatments for acute respiratory distress syndrome. Crit Care Med. 2002;30(8):1772-1777.

41. Lee WL, Slutsky AS. Sepsis and endothelial permeability. NEngl J Med. 2010;363(7):689-691.

42. Goldenberg NM, Steinberg BE, Slutsky AS, Lee WL. Broken barriers: a new take on sepsis pathogenesis. Sci Transl Med. 2011;3(88):88ps25.

43. Shapiro NI, et al. The association of endothelial cell signaling, severity of illness, and organ dysfunction in sepsis. Crit Care. 2010;14(5):R182.

44. Sessler CN, et al. Circulating ICAM-1 is increased in septic shock. Am J Respir Crit Care Med. 1995;151(5):1420-1427.

45. Mutunga M, et al. Circulating endothelial cells in patients with septic shock. Am J Respir Crit Care Med. 2001;163(1):195-200.

46. Shapiro NI, Yano K, Sorasaki M, Fischer C, Shih SC, Aird WC. Skin biopsies demonstrate site-specific endothelial activation in mouse models of sepsis. J Vasc Res. 2009;46(5):495-502.

47. London NR, et al. Targeting Robo4-dependent Slit signaling to survive the cytokine storm in sepsis and influenza. Sci Transl Med. 2010;2(23):23ra19.

48. Gill SE, Taneja R, Rohan M, Wang L, Mehta S. Pulmonary microvascular albumin leak is associated with endothelial cell death in murine sepsis-induced lung injury in vivo. PLoS One. 2014;9(2):e88501.

49. Matthay MA, Zemans RL. The acute respiratory distress syndrome: pathogenesis and treatment. Annu Rev Pathol. 2011;6:147-163

50. Millar FR, Summers C, Griffiths MJ, Toshner MR, Proudfoot AG. The pulmonary endothelium in acute respiratory distress syndrome: insights and therapeutic opportunities. Thorax. 2016;71(5):462-473.

51. Maeda N, Yang F, Barnett DR, Bowman BH, Smithies O. Duplication within the haptoglobin Hp2 gene. Nature. 1984;309(5964):131-135.

52. Smithies O. Grouped variations in the occurrence of new protein components in normal human serum. Nature. 1955;175(4450):307-308.

53. Smithies O, Walker NF. Genetic control of some serum proteins in normal humans. Nature. 1955;176(4496):1265-1266.

54. Asleh R, et al. Genetically determined heterogeneity in hemoglobin scavenging and susceptibility to diabetic cardiovascular disease. Circ Res. 2003;92(11):1193-1200.

55. Janz DR, Ware LB. The role of red blood cells and cell-free hemoglobin in the pathogenesis of ARDS. J Intensive Care. 2015;3:20.

56. Shi X, et al. Haptoglobin 2-2 genotype is associated with increased risk of type 2 diabetes mellitus in northern Chinese. Genet Test Mol Biomarkers. 2012;16(6):563-568.

57. Japan Blood Products Organization. Human haptoglobin general product information overview. https://www.jbpo.or.jp/med/ di/file/6451/. Accessed October 4, 2019 
58. Yamamoto H, Nishikawa S, Yamazaki K, Kudo R. Efficacy of haptoglobin administration in the early postoperative course of patients with a diagnosis of HELLP syndrome. J Obstet Gynaecol. 2000;20(6):610-611.

59. Hashimoto K, Nomura K, Nakano M, Sasaki T, Kurosawa H. Pharmacological intervention for renal protection during cardiopulmonary bypass. Heart Vessels. 1993;8(4):203-210.

60. Kanamori Y, et al. [The effects of administration of haptoglobin for hemolysis by extracorporeal circulation]. Rinsho Kyobu Geka. 1989;9(5):463-467.

61. Gando S, Tedo I. The effects of massive transfusion and haptoglobin therapy on hemolysis in trauma patients. Surg Today. 1994;24(9):785-790.

62. Boretti FS, et al. Sequestration of extracellular hemoglobin within a haptoglobin complex decreases its hypertensive and oxidative effects in dogs and guinea pigs. J Clin Invest. 2009;119(8):2271-2280.

63. Graw JA, et al. Haptoglobin or hemopexin therapy prevents acute adverse effects of resuscitation after prolonged storage of red cells. Circulation. 2016;134(13):945-960.

64. Baek JH, Zhang X, Williams MC, Schaer DJ, Buehler PW, D’Agnillo F. Extracellular Hb enhances cardiac toxicity in endotoxemic guinea pigs: protective role of haptoglobin. Toxins (Basel). 2014;6(4):1244-1259.

65. Remy KE, et al. Haptoglobin improves shock, lung injury, and survival in canine pneumonia. JCI Insight. 2018;3(18):123013.

66. Levy AP, et al. Haptoglobin genotype is a determinant of iron, lipid peroxidation, and macrophage accumulation in the atherosclerotic plaque. Arterioscler Thromb Vasc Biol. 2007;27(1):134-140.

67. Pradilla G, et al. Systemic L-citrulline prevents cerebral vasospasm in haptoglobin 2-2 transgenic mice after subarachnoid hemorrhage. Neurosurgery. 2012;70(3):747-756.

68. Chaichana KL, Levy AP, Miller-Lotan R, Shakur S, Tamargo RJ. Haptoglobin 2-2 genotype determines chronic vasospasm after experimental subarachnoid hemorrhage. Stroke. 2007;38(12):3266-3271.

69. O'Neal HR, et al. Prehospital statin and aspirin use and the prevalence of severe sepsis and acute lung injury/acute respiratory distress syndrome. Crit Care Med. 2011;39(6):1343-1350.

70. Kelly KJ, Sandoval RM, Dunn KW, Molitoris BA, Dagher PC. A novel method to determine specificity and sensitivity of the TUNEL reaction in the quantitation of apoptosis. Am J Physiol, Cell Physiol. 2003;284(5):C1309-C1318.

71. Reiter CD, et al. Cell-free hemoglobin limits nitric oxide bioavailability in sickle-cell disease. Nat Med. 2002;8(12):1383-1389.

72. Brittain EL, et al. Elevation of plasma cell-free hemoglobin in pulmonary arterial hypertension. Chest. 2014;146(6):1478-1485

73. Janz DR, et al. Longer storage duration of red blood cells is associated with an increased risk of acute lung injury in patients with sepsis. Ann Intensive Care. 2013;3(1):33.

74. Azarov I, et al. Rate of nitric oxide scavenging by hemoglobin bound to haptoglobin. Nitric Oxide. 2008;18(4):296-302.

75. Levy MM, et al. 2001 SCCM/ESICM/ACCP/ATS/SIS International Sepsis Definitions Conference. Intensive Care Med. 2003;29(4):530-538

76. Singer M, et al. The Third International Consensus Definitions for Sepsis and Septic Shock (Sepsis-3). JAMA. 2016;315(8):801-810.

77. Wynn JL, et al. Increased mortality and altered immunity in neonatal sepsis produced by generalized peritonitis. Shock. 2007;28(6):675-683.

78. Wynn JL, et al. Defective innate immunity predisposes murine neonates to poor sepsis outcome but is reversed by TLR agonists. Blood. 2008;112(5):1750-1758.

79. Shaver CM, et al. Cell-free hemoglobin augments acute kidney injury during experimental sepsis. Am J Physiol Renal Physiol. 2019;317(4):F922-F929.

80. Bastarache JA, et al. Low levels of tissue factor lead to alveolar haemorrhage, potentiating murine acute lung injury and oxidative stress. Thorax. 2012;67(12):1032-1039.

81. Blackwell TS, Lancaster LH, Blackwell TR, Venkatakrishnan A, Christman JW. Chemotactic gradients predict neutrophilic alveolitis in endotoxin-treated rats. Am J Respir Crit Care Med. 1999;159(5 Pt 1):1644-1652.

82. Ferguson ND, et al. The Berlin definition of ARDS: an expanded rationale, justification, and supplementary material. Intensive Care Med. 2012;38(10):1573-1582.

83. Janz DR, et al. Association between haptoglobin, hemopexin and mortality in adults with sepsis. Crit Care. 2013;17(6):R272

84. Soejima M, Koda Y. TaqMan-based real-time PCR for genotyping common polymorphisms of haptoglobin (HP1 and HP2). Clin Chem. 2008;54(11):1908-1913.

85. Koch W, et al. Genotyping of the common haptoglobin Hp 1/2 polymorphism based on PCR. Clin Chem. 2002;48(9):1377-1382.

86. Zhao B, et al. A genome-wide association study to identify single-nucleotide polymorphisms for acute kidney injury. Am $J$ Respir Crit Care Med. 2017;195(4):482-490.

87. Browning SR, Browning BL. Rapid and accurate haplotype phasing and missing-data inference for whole-genome association studies by use of localized haplotype clustering. Am J Hum Genet. 2007;81(5):1084-1097.

88. 1000 Genomes Project Consortium, et al. A global reference for human genetic variation. Nature. 2015;526(7571):68-74

89. International HapMap Consortium. A haplotype map of the human genome. Nature. 2005;437(7063):1299-1320.

90. Knaus WA, Draper EA, Wagner DP, Zimmerman JE. APACHE II: a severity of disease classification system. Crit Care Med. $1985 ; 13(10): 818-829$.

91. R Core Team. R: A language and environment for statistical computing. R Foundation for Statistical Computing. http:// www.R-project.org/. Accessed October 4, 2019.

92. Signorell A. DescTools: tools for descriptive statistics. R-Project. https://cran.r-project.org/web/packages/DescTools/index.html. Accessed October 4, 2019.

93. Vanderbilt University Department of Biostatistics. rms: regression modeling strategies. Vanderbilt University. http://biostat. mc.vanderbilt.edu/wiki/Main/Rrms. Accessed October 4, 2019.

94. RStudio Team. RStudio: integrated development environment for R. https://rstudio.com/. Accessed October 4, 2019.

95. Wickham H. ggplot2: elegant graphics for data analysis. 2nd ed. New York, New York, USA; Springer: 2016. 